\title{
Illuminating the future with LEDs
}

\section{Silvino Jose Antuña Presa}

School of Electrical and Electronic Engineering and Tyndall National Institute, UCC

Knowledge once gained casts a light beyond its own immediate boundaries. (John Tyndall)

\section{Introduction}

Light-emitting diodes also known as LEDs are all around us: they are used in TVs, computers, mobile phones, street lighting and even in our homes in the form of new energy saving and long lifespan lamps.

Now you might be wondering: "why all the fuss? Is this another 'new thing' that is going to disappear in a couple of years or is this something that is going to stay?" Well, to the truth, LEDs have been around for some time: they were initially used as small indicator lamps and for small displays; in fact, almost any appliance you may have at home will have a small LED lamp to tell you whether it is on or off.

Things started to change when the first blue LED was demonstrated in 1994. Before this achievement the only available colours for LEDs with decent efficiency were red and orange-red. This was good for indicators and small displays but not for general lighting applications because we cannot convert red light efficiently into white light. However, we can readily convert violet and blue light into white light using yellow and red phosphors. So once the blue LED became available, we could start to develop white LED sources and the possibility of replacing our old lamps by new more efficient ones became real.

Just a couple of years later first green LEDs appeared on the scene. Green light sources are very important for two main reasons: firstly, because tri-chromatic red-green-blue light is needed for full colour displays, such as a computer or TV screens. Secondly, because the human eye has greater response to green and yellow light. This characteristic of the eye allows us to observe green light sources as being brighter, even if they are not as powerful as light sources in other colours.

\section{Let there be light: How light-emitting diodes work.}

\section{Traditional light sources}

Traditional light sources use heat to generate light. For example, the common incandescent bulb uses electricity to heat a small filament-wire. When this filament is hot enough 
it starts to emit visible light; the physical effect of getting light from a hot body is called incandescence, which is a special case of thermal radiation or in other words, emission of electromagnetic radiation from a hot surface.

As we all know from personal experience, if you touch an incandescent bulb after it has been on for a while, it is hot. That is because not all the electricity driven to the bulb is converted into light; in fact, the largest part of the electricity is converted into heat. This is not an optimal solution because we want to get as much light as possible from as little power as possible. In other words, what we want is high efficiency light sources which transform current into light and not into heat.

Fluorescent lamps are a more efficient alternative although with several drawbacks: There is still a significant portion of current being converted into heat, and the generated light is ultraviolet which can be harmful and requires conversion to white light using a phosphor coating inside the fluorescent bulb. However, the biggest problem is that fluorescent lamps contain mercury, which is highly toxic and harmful to the environment. So in general fluorescent lamps are a good solution and still widely used for example in office buildings. However; we still need to search for a better solution.

\section{Semiconductor light sources}

Luckily enough, there is another way of getting light without the heat by using semiconductor materials. A semiconductor is a special kind of material which only conducts electrical current under certain conditions but not others. This allows us to control very precisely the electrical current through the material and which is the foundation of modern electronics. In a pure semiconductor at room temperature we can find two kinds of particles able to move when applying a bias. The negatively charged particles are electrons and the positive ones are holes, and both are called carriers in general. In a pure semiconductor material we have the same number of electrons and holes but we can add different types of impurities to these pure semiconductors and change its properties, this is called semiconductor doping. If we dope a material to have more holes than electrons, we end up with a material known as a (positive) $p$-type semiconductor and conversely, if we end up with more electrons than holes we obtain a (negative) $n$-type semiconductor. Furthermore, we can join a $p$-type and an $n$-type semiconductor in what is called a $p$ - $n$ junction. This forms a device which on one side has a $p$-type layer with more holes than electrons, then a junction-layer which is depleted of any carriers and finally a $n$-type layer with more electrons than holes. This kind of device is called a diode and it is widely used in electronics.

Ok, we now know what a diode is; but how do we get light from a semiconductor? If we look inside the material, we find that electrons and holes in the semiconductor have different energy levels with electrons in the higher energy states, and holes in the lower ones. When an electron meets a hole they are attracted due to their opposite charges; they 
can recombine, in which case both disappear, and the energy is released as light. This is the key to understanding why LEDs can be so efficient: In an ideal LED, all the electrical energy can be converted into light without emitting any heat.

So when a LED is connected to a power supply, electrons and holes from the $n$ - and $p$-side both move towards the junction where they recombine. And because we select a material with a suitable separation between the energy levels, we get light emission in the colour that corresponds with the chosen energy gap.

Figure 1 gives an example of the efficiency of incandescent, fluorescent and LED lamps. Incandescent lamps and the more recent halogen lamps are widely used in our homes but they are clearly the ones with lower light outputs per Watt. As mentioned before, fluorescent lamps have a good efficiency, but they have problems related with harmful UVemissions and toxicity. On the right hand side of the graph the recent progress on LEDs is shown: current LEDs now surpass even the best fluorescent lamps.

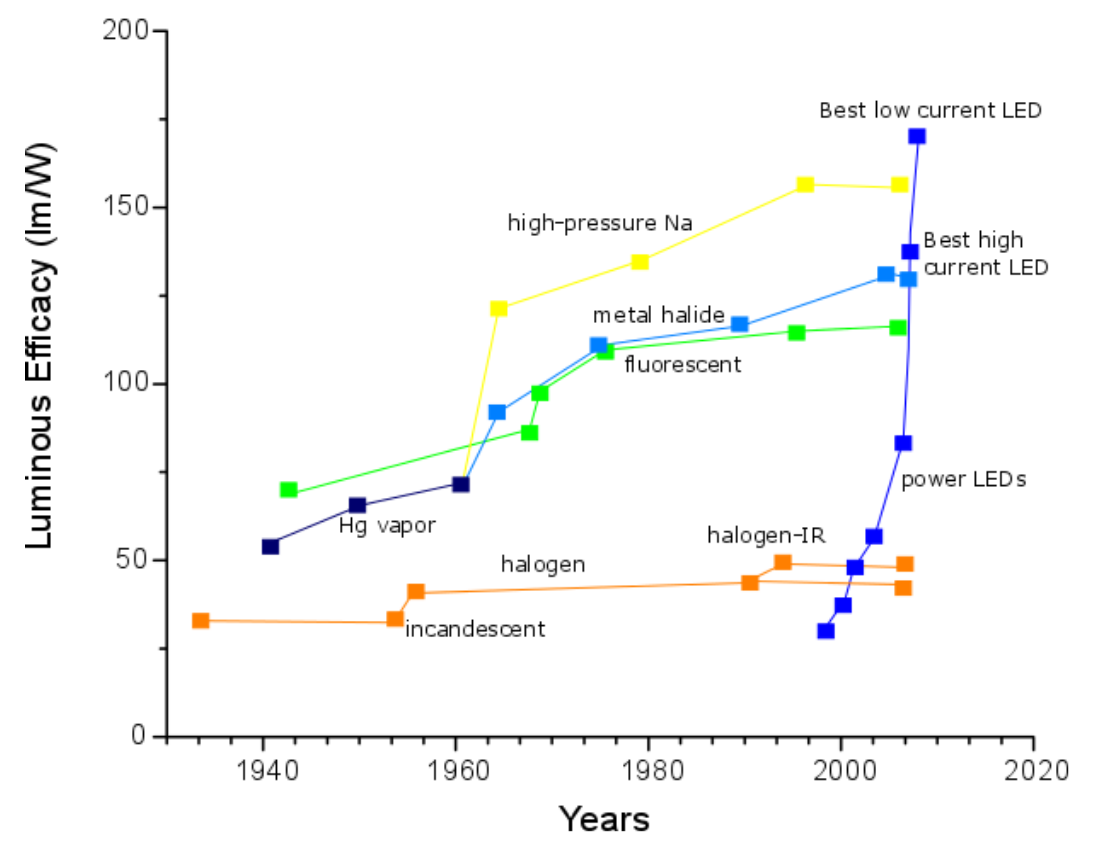

Figure 1: Conversion efficiencies of different light sources. Source: Author

\section{A material worth a Nobel prize: Gallium nitride}

The Nobel Prize for physics was awarded in 2014 to I. Akasaki, H. Amano and S. Nakamura "for the invention of efficient blue light-emitting diodes which has enabled bright and energy-saving white light sources". As explained in the introduction, a blue light source is required to achieve white light by conversion of part of its light output with yellow and red phosphors or combining it with red and green light sources. These first LEDs were fabricated using a material called gallium nitride ( $\mathrm{GaN})$. $\mathrm{GaN}$ is a semiconductor capable of emitting light in violet and blue colours, but what is interesting is the fact that if we 
add small percentages of the chemical element indium to the GaN, we can get lower energy colours such as green and yellow and theoretically reach even orange and red, thus covering the entire visible spectrum.

The problem is that this material is very complex to work with. In fact, even before the first demonstration of the blue LED it was known that a blue LED could be fabricated with this material, but it was very difficult to achieve good p-type GaN material. The 2014 Nobel laureates were able to overcome this difficulty and since the early 1990s the capability of growing high quality GaN LED material has been demonstrated and it has become feasible to fabricate LEDs in a commercially viable way.

We can summarize the other main advantages of gallium nitride as:

$\square$ It has a good thermal conductivity, leading to simpler packaging with a reduction in system volume and weight.

$\square$ It operates at a lower supply voltage, and at a lower temperature and with increased light output.

$\square$ It has a faster switching speed and reduced electrical noise. This allows for the use of LEDs in optical communication systems.

$\square$ The materials are environmentally friendly, and no hazardous materials are required for the fabrication of devices.

$\square$ The material is robust, which results in long lifespan devices.

\section{My research on GaN-based light emitting diodes}

At this point it is clear that LEDs provide a nice solution to illumination problems and we have pointed out some of the unique properties of gallium nitride. Nevertheless, there are many things still to be researched: Gallium nitride is a complex material and some of the light generating mechanisms are still poorly understood. In fact, some of them have been the subject of debate for more than a decade and we still do not have conclusive answers.

On the other hand, we are able to produce efficient sources of white light because blue GaN-based LEDs have energy efficiencies of up to $85 \%$. However, this is not true for green GaN-based LEDs where the highest efficiency is presently around 30\%. If we move further towards yellow, and orange the efficiencies are even lower and no gallium nitride based devices are commercially available yet in these colours.

So, the main goal of my research is to improve the understanding of what makes this material emit light and how to maximize the emission efficiency in all the visible colours. A combination of electrical and optical excitation is used to get insight into the recombination processes of electrons and holes inside these materials. Also, different semiconductor growth techniques are compared to see the advantages and disadvantages of the various 


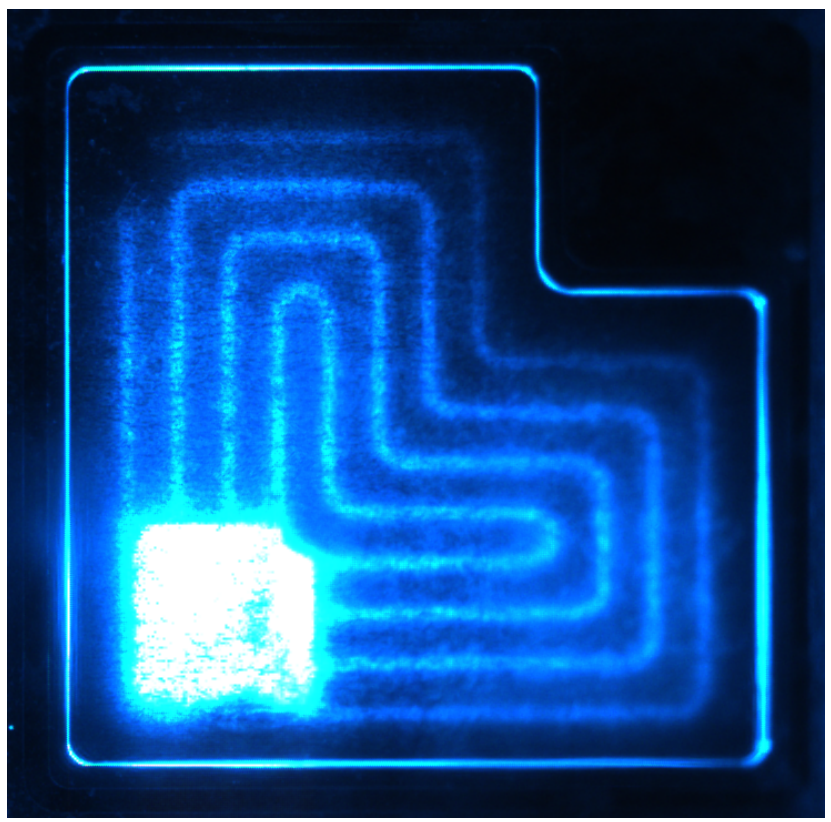

Figure 2: Microscope image of light output under electrical excitation for a blue LED fabricated at Tyndall.

crystallographic structures of GaN. With the aid of the processing facilities at Tyndall we can also investigate the effect of the device shape and the fabrication steps on the light emission of processed LEDs as illustrated in Figure 2.

Source: Author

\section{Final words}

We are just at the beginning of the LED era for general illumination. The knowledge developed in semiconductor fabrication and packaging is allowing us to fabricate sciencefiction types of devices like flexible, miniature displays, holograms, virtual and augmented reality helmets and smart lamps which can change the intensity, colour, and direction of the light depending on the needs of the user. All of this is made possible by advances in semiconductor lighting technology and microfabrication processes.

A lot of work still needs to be done though. A better understanding is required to see what happens inside materials such as GaN to achieve even better light sources to illuminate our future.

I would like to thank my supervisors Pleun Maaskant and Brian Corbett and all the people from Tyndall National Institute for their support during my research, especially to the people belonging to the GaN group. I also would like to acknowledge funding by the European Community's 7th Framework Programme. 